\title{
Node Localization In Wireless Sensor Networks By Randomly Generated Tokens
}

\author{
Ejaz Hussain ${ }^{1}$, Xiong Zhang ${ }^{1}$, Chao $\mathrm{Li}^{1}$ and Sadique Ahmed Bugti ${ }^{2}$ \\ ${ }^{1}$ School of Computer Science and Engineering \\ Beihang University, Beijing, China \\ ${ }^{2}$ Balochistan University of Information Technology, Pakistan
}

\begin{abstract}
Wireless sensor network is a type of communication which gives a steadfast communication at the minimal expense. This paper highlights one of the fundamental concern namely node localization by using the combined effect of randomly generated tokens at anchor nodes and vector iterative process (RGT_VIP). Time synchronization is a critical aspect in the wireless sensor networks for their data transmission. But with this approach, we can range and estimate the position of deployed nodes without depending on central synchronized time reference at receivers end and at the anchors/transmitter end. Results show that this newly proposed method gives a greater accuracy in terms of average distance error on different variance of Gaussian values and performs better than synergetic localization algorithm on higher values of variance.
\end{abstract}

Keywords-Node localization; Randomly generated tokens; WSN; Ad hoc sensor networks

\section{INTRODUCTION}

It is evident from the recent years that there is an increased interest in the use of wireless sensor networks (WSNs) in numerous applications such as forest monitoring, disaster management, space exploration, factory automation, border protection and battlefield surveillance [1,2]. The burning issue in wireless sensor networks is the automatic localization of the deployed nodes. A number of research activities have been carried out in this field and the same way a large number of algorithms have been presented so far. These algorithms have their range from simple to very complex, but they all only categories into two different fields namely range based methods and range free methods [3].

Range free methods cannot provide the location of deployed nodes so accurate but they are cost effective and robust to noise because the only requirement for such systems is connectivity and number of hops between nodes. Niculescu and Nath proposed a typical range-free algorithm DV-HOP [4]. DV-HOP uses distance-vector forwarding technique to get the minimum hop count from a node to heard anchors. In this method, deployed nodes estimate their positions by using the triangulation method and with the help of corrective measures by anchors. There are some other methods with better accuracy but at the cost of complicated circuitry. Multidimensional scaling maps (MDS-MAP) was proposed by Sheng et al. [5] and after this variance of MDS-MAP are presented as centralized $\operatorname{MDS}-\operatorname{MAP}(\mathrm{C})$ and distributed
MDS-MAP (P). There is another method Support Vector Machine (SVM) proposed by Tran and Nguyen [6]. In this method machine learning approach is used to localize the nodes.

In range-based methods, range measurements are used, such as received signal strength (RSS) [7], it is the voltage measured at receivers. RSS measurements are relatively inexpensive and simple in implementation. Much of the work is being done on this topic, yet it is notoriously unpredictable [8]. Also before using this approach into a very critical application, their sources of errors must be well understood. Angle of arrival (AOA) [9] provides localization information complementary to the Time of arrival (TOA) and RSS measurements. Two popular ways are used to measure the AOA, the first one used array processing technique and in second approach, AOA estimation uses RSS ratio between two (or more) directional antennas.

Time difference of arrival (TDOA) [10] measurements measure the difference between the arrival times of a transmitter signal at two receivers respectively. In $\Re^{2}$, denote the coordinates of the two receivers by $X_{i} \& X_{j}$, and the coordinates of the transmitter by $X_{t}$. The measured TDOA is related to the locations of the two receivers by

$$
\Delta t_{i j}=t_{i}-t_{j}
$$

Where $t_{i}$ and $t_{j}$ are the arrival times of the transmitter signal at receivers $\mathrm{i}$ and $\mathrm{j}$ respectively. The popular distance estimation method is the TOA [11] method in which the range is estimated based on the time the signal spends traveling from the transmitter to the receiver. Since it is very much obvious that, the speed of RF propagation is very well known in both free space and air, it gives a direct estimation of the distance between the transmitter and the receiver once the travel time is estimated. The basic equation needed to obtain the distance is given as

$$
d=\tau C
$$

Where $d$ is the distance estimate $\tau$ is the TOA of signal and $\mathrm{c}$ is the speed of light. In a distributed environment such as wireless sensor networks there may be no central synchronized time reference that regulates the activities of each node. Instead, each node manages its own time reference. Accurate TOA estimation needs perfect synchronization 
between the clocks of the transmitter and the receiver. Clock synchronization might be achieved by regular data exchange between the transmitter and the receiver or an additional anchor for correcting the clock bias. Although three anchors are necessary to obtain position, a fourth anchor will be needed for time correction. Many other localization algorithms like some in [12-15] have been developed for the evaluation of positioning of nodes in the wireless sensor networks.

This paper highlights one of the fundamental concern namely node localization by using randomly generated tokens at anchor nodes and vector iterative process. With this approach, we can range and estimate the position of deployed nodes without depending on central synchronized time reference at receivers end and at the anchors/transmitter end.

The rest of the paper is organized as follows. Section II discusses the motivation for developing RGT_VIP. Proposed approach is introduced in section III. Simulation results and conclusion are presented in section IV and V respectively.

\section{MOtIVATION FOR RGT_VIP}

From the last decade, wireless communication emerged as the biggest engineering success not only in research and development but also in the market size and impact on the society. Wireless computer networks, wireless positioning system and wireless sensor networks, are few of names here; they have changed the composition of their application and most importantly have created new directions of research in communication system fields. In WSN, there are many issues and one burning issue is the automatic localization of the deployed nodes. So, in this paper we focus on method which gives reliable positioning information of the deployed nodes before sending the data to the destinations. For this, only information we need is, the positioning of the anchor/beacons nodes and the randomly generated tokens from the same.

\section{PROPOSED APPROACH}

We use MATLAB platform environment for the simulation of proposed method. $100 \mathrm{~m}^{2}$ area is considered for the deployed infrastructure. Fig.1 shows the layout of randomly distributed nodes in the area of concern.

We placed three anchor nodes at the three corners of the square for the basic communication. Coordinates of the anchor nodes are shown in the Fig 1 which are A $(0,0), \mathrm{B}(0$, $100)$ and $C(100,100)$. The goal of this paper is to locate and estimate the position of $\mathrm{N}$ randomly distributed nodes in the bounded region without depending on central synchronized time reference at receivers end and at the anchors/transmitter end.

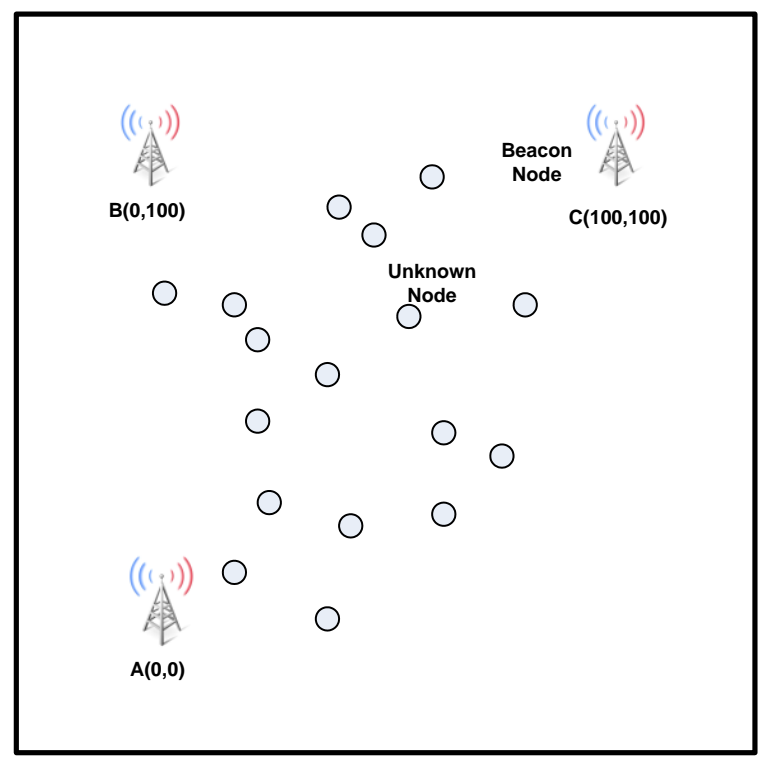

Figure 1. Layout diagram of deployed nodes

To address the need of nodes deployment in wireless sensor network in distributed environment, we used an algorithm named as RGT_VIP, and to illustrate the feasibility of this algorithm a general assumption is followed as all $\mathrm{N}$ nodes at any position can hear the beacons of all anchor nodes.

Below is the process of how we can generate the token and how to send the position and token information to other nodes.

\section{A. Token Generation Process}

Calculations necessary for the measurement of the distance between anchor nodes and token traveling timings are covered in this stage. This stage is also responsible for the calculation of time difference values of the calculated vectors.

An estimated value of vector $\mathrm{AX}$ is used to determine the values of rest of vectors BX \& CX by simply vectors head to tail rule. With the help of these estimated values, we can estimate the time difference values. These time difference values are then compared to the values with the calculated ones in the first stage. Co- ordinates of unknown nodes can be determined if the resultant of two values is equal to zero, otherwise a new estimated value is assigned to vector AX. The process is repeated until we get the diff nearly equal to zero.

For generation of tokens, a 2-byte (16-bits) length of a data segment is sent periodically from anchor nodes for building the basic concept of this work. The detail of the 16 bits is depicted in Fig. 2

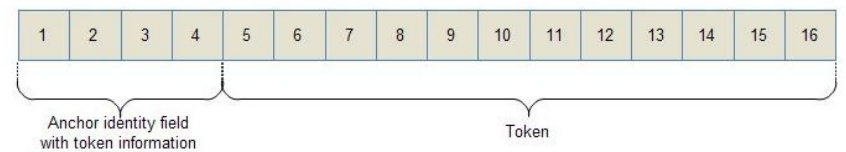

Figure 2. 16-bit data segment for token generation process

1) Sending the token information 
The first 4 bits (shown in Fig 2, 3 and tabulated in Table 1) tell us the anchor identity with address/token information. The detail of these bits is as under.

a) Bits (1-2) represents the anchor identity.

00 represent the data from anchor node $\mathrm{A}$

10 represent the data from anchor node $\mathrm{B}$

11 represent the data from anchor node $\mathrm{C}$

b) Bit (3-4) distinguishes the address or token sent from the anchor nodes

00 Address sent from anchor nodes

01 Token from anchor nodes $\mathrm{A}$ and $\mathrm{C}$

10 Node's " $A$ " token sent from anchor node B

11 Node's " $C$ " token sent from anchor node B

c) Bits (5-16), these 12 bits give us the value for the randomly generated tokens.

2) Sending the Address information

The first 4 bits are same as detail provided in A1. Bit- 5 gives the axis position information, 0 for $\mathrm{X}$-Axis and 1 for Y-Axis. For bits 6-16, these eleven bits give the value of $\mathrm{X}$ -axis or Y-axis. Thus each anchor node can send the coordinate value of either $X$ or $Y$ axis from 0 2047. Fig 3 shown below is depicted to present this scenario.

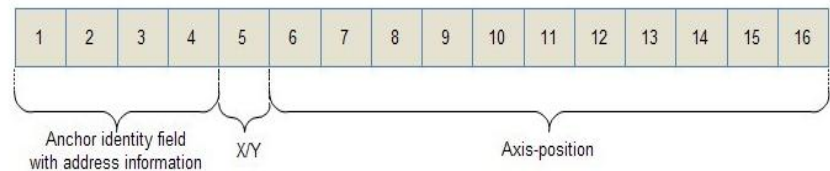

Figure 3. 16-bit data segment for sending position information

A self explanatory Table1 below shows the overall picture for the address and token management system, and in Table2 notation and their meanings are presented.

TABLE I. Distinguish BETWEen ADDRESS AND TOKEN

\begin{tabular}{|c|c|c|c|l|}
\hline 0 & 0 & 0 & 0 & Address sent from Anchor node A \\
\hline 0 & 0 & 0 & 1 & Token sent from Anchor node A \\
\hline
\end{tabular}

\begin{tabular}{|l|l|l|l|l|}
\hline 1 & 1 & 0 & 0 & Address sent from Anchor node $\mathrm{C}$ \\
\hline 1 & 1 & 0 & 1 & Token sent from Anchor node C \\
\hline
\end{tabular}

\begin{tabular}{|c|c|c|c|l|}
\hline 1 & 0 & 0 & 0 & Address sent from Anchor node B \\
\hline 1 & 0 & 1 & 0 & Node's A Token sent from Anchor node B \\
\hline 1 & 0 & 1 & 1 & Node's C Token sent from Anchor node B \\
\hline
\end{tabular}

TABLE II. NOTATIONS AND THEIR MEANINGS

\begin{tabular}{|c|c|}
\hline Notation & Meaning \\
\hline$\Delta T_{1}$ & $\begin{array}{l}\text { Time difference of signal transmitted from Node A to X directly } \\
\text { and from A to X via B }\end{array}$ \\
\hline$\Delta T_{2}$ & $\begin{array}{l}\text { Time difference of signal transmitted from Node } \mathrm{C} \text { to } \mathrm{X} \text { directly } \\
\text { and from } \mathrm{C} \text { to } \mathrm{X} \text { via } \mathrm{B} \text {. }\end{array}$ \\
\hline$T_{1(\text { start })}$ & $\begin{array}{l}\text { Timer } 1 \text { at every un-known node starts upon receiving the signal } \\
\text { from beacon node A }\end{array}$ \\
\hline$T_{1(\text { stop })}$ & $\begin{array}{l}\text { Timer } 1 \text { at every un-known node stops upon receiving the signal } \\
\text { from beacon node B carrying the token of node A }\end{array}$ \\
\hline$T_{2(\text { start })}$ & $\begin{array}{l}\text { Timer } 2 \text { at every un-known node starts upon receiving the signal } \\
\text { from beacon node } \mathrm{C}\end{array}$ \\
\hline$T_{2(\text { stop })}$ & $\begin{array}{l}\text { Timer } 2 \text { at every un-known node stops upon receiving the signal } \\
\text { from beacon node B carrying the token of node C }\end{array}$ \\
\hline $\mathrm{AB}_{(t)}$ & Time it takes the signal to propagate from node A to Node B \\
\hline$A X_{(t)}$ & $\begin{array}{l}\text { Time it takes the signal to propagate from node A to un-known } \\
\text { node } \mathrm{X}\end{array}$ \\
\hline$B X_{(t)}$ & $\begin{array}{l}\text { Time it takes the signal to propagate from node B to un-known } \\
\text { node } \mathrm{X}\end{array}$ \\
\hline$C X_{(t)}$ & $\begin{array}{l}\text { Time it takes the signal to propagate from node } \mathrm{C} \text { to un-known } \\
\text { node } \mathrm{X}\end{array}$ \\
\hline$C \mathrm{~B}_{(t)}$ & Time it takes the signal to propagate from node $\mathrm{C}$ to Node $\mathrm{B}$ \\
\hline$S_{P A}$ & Positioning signal transmitted from Node A \\
\hline$S_{P C}$ & Positioning signal transmitted from Node C \\
\hline$S_{P B}$ & Positioning signal transmitted from Node B \\
\hline$S_{T A}$ & Token signal transmitted from node A \\
\hline$S_{T C}$ & Token signal transmitted from node $\mathrm{C}$ \\
\hline$S_{T B A}$ & Token signal transmitted from node B carrying token of A \\
\hline$S_{T B C}$ & Token signal transmitted from node B carrying token of C \\
\hline
\end{tabular}

These is a mechanism which tell the anchor nodes what to transmit so that the deployed nodes workout to estimate their position. In our algorithm, the function of anchor nodes is to periodically transmit their position information as well as randomly generated tokens. We established the timing sequence as shown in Fig.4 and on the basis of this sequence; anchor nodes (A, C and B) transmit their information. 

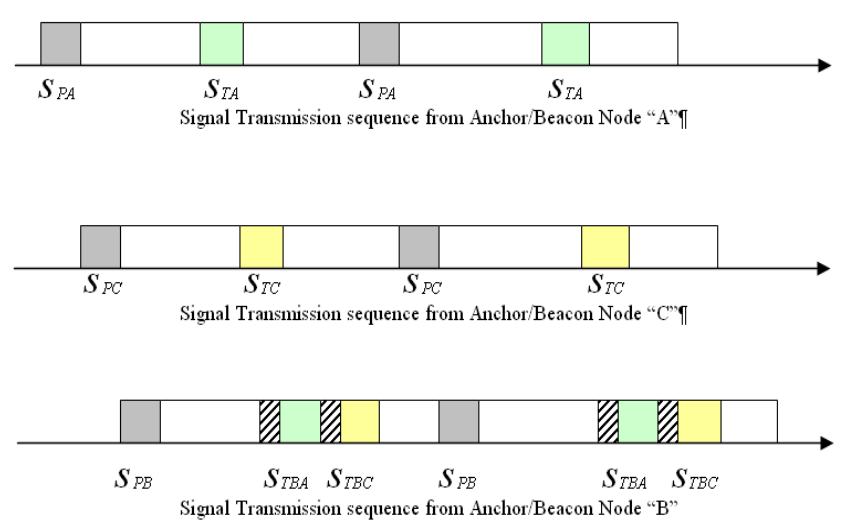

Figure 4. Signal transmission sequence from Anchor nodes

4) Measuring the distance and traveling time of signal between anchor nodes

At the start anchor nodes periodically send their position information (SPA, SPB and SPC) along with their identity. The signal disseminates to all unknown nodes and anchor nodes, but all three anchor nodes ignore the received positioning signals. The deployed nodes in the region calculate the distances between anchors nodes from the available position information. Euclidean distance between two anchor nodes is given below..

$$
d t(p, q)=\sqrt{\left(p_{1}-q_{1}\right)^{2}+\left(p_{2}-q_{2}\right)^{2}}
$$

From the available value of distance between two points, signal propagation time can be calculated as under .

$$
t(p, q)=C / d t(p, q)
$$

\section{5) Constructing the time difference equations}

In this scheme anchor nodes send periodically randomly generated tokens with their identity. In this paper, we chooses two anchor nodes A and C to send these tokens and anchor B to re-transmit the same after receiving from either $\mathrm{A}$ or $\mathrm{C}$ but with its own identity.

When Token disseminated form A, it simultaneously propagates to anchor nodes $\mathrm{B} \& \mathrm{C}$ as well as to all $\mathrm{N}$ unknown nodes. Timer $\mathrm{T} 1$ at unknown nodes starts upon receiving of token from A and stops on the receiving the same from B. Time duration between these arrivals of token is calculated as follows.

$$
\Delta T_{1}=T_{1(\text { stop })}-T_{1(\text { start })}
$$

Similarly, when the token signal disseminated form C, received at all unknown nodes, T2 starts and upon receiving the same from anchor node B, it stops. Difference of this time equation is given below.

$$
\Delta T_{2}=T_{2(\text { stop })}-T_{2(\text { start })}
$$

Equations (3) and (4) can be re-written as under

$$
\begin{gathered}
\Delta \mathrm{T}_{1}=\mathrm{AB}_{(t)}+B X_{(t)}-A X_{(t)} \\
\Delta \mathrm{T}_{2}=C \mathrm{~B}_{(t)}+B X_{(t)}-C X_{(t)}
\end{gathered}
$$

So by knowing the signal propagation time between $\mathrm{AB}$ and $\Delta T_{1}$, we can get the equation of time difference for $\mathrm{BX}$ and $\mathrm{AX}$.

$$
B X_{(t)}-A X_{(t)}=\Delta \mathrm{T}_{1}-\mathrm{AB}_{(t)}
$$

Similarly knowing the signal propagation time between $\mathrm{CB}$ and $\Delta T_{2}$, we can get the equation of time difference for BX and $\mathrm{CX}$.

$$
B X_{(t)}-C X_{(t)}=\Delta \mathrm{T}_{2}-C \mathrm{~B}_{(t)}
$$

The contribution of (7) and (8) is very important for the further calculations.

\section{B. Vector Iterative Process}

In VIP stage, we determine the coordinates of unknown nodes by simple vector manipulation. We used an iterative method along with geometrical computation for getting the desired results. An iterative method is a mathematical procedure that generates a sequence of improved approximate solutions for a class of problems. An iterative process should be convergent, i.e., it should come closer to the desired result as the number of iterations increases.

We divide the Vector Manipulation iteration process into two steps to make things clear.

\section{1) Random assignment values}

Any unknown node say $X_{i}$ assign a random value to vector $A X$ with respect to anchor node $A$. This node has already the value of $\overrightarrow{A B}$ calculated earlier in 3(A.1). Vector $A X$ with slope $\theta$ is shown Fig 5 .

Now by knowing the values of $\overrightarrow{A B}$ and $A X$, length and slope of $X B$ can be calculated by head to tail rule.

$$
\overrightarrow{X B}=\overrightarrow{A B}-\overrightarrow{A X}
$$

Similarly, the length and slope of $C X$ can be determined by the following equation.

$$
\overrightarrow{C X}=\overrightarrow{C B}-\overrightarrow{X B}
$$


Therefore, once we have the values of $\mathrm{AX}, \mathrm{XB}$ and $\mathrm{CX}$, we can easily determine the signal propagation time between them as well as the signal propagation time difference between $\mathrm{BX} \& \mathrm{AX}$ and $\mathrm{BC} \& \mathrm{CX}$.

\section{2) Refinement process}

In this step a refinement process takes place, measured time values from equation (3) and (4) are compared with the estimated values calculated in the step-1. If the difference between all the corresponding values is equal to zero the iteration/refinement process stop and equations (11) and (12) gives the coordinates of $X_{i}$, otherwise new value is assigned to $A X$ till we achieve the refined desired result.

$$
\begin{gathered}
X_{i(x-a x i s)}=\left[d / \operatorname{sqrt}\left(n^{2}+1\right)\right]+A_{x} \\
X_{i(y-a x i s)}=d\left[\operatorname{sqrt}\left(n^{2}\right) / \operatorname{sqrt}\left(n^{2}+1\right)\right]+A_{y}
\end{gathered}
$$

In the above equations $\mathrm{d}$ and $\mathrm{n}$ are the assigned distance and slope to vector $A X$.

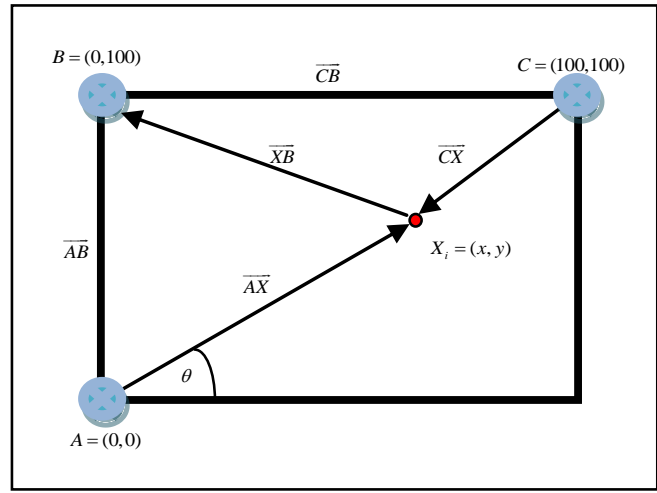

Figure 5. Vector Manipulation for Node X

\section{SIMULATION RESULTS}

Accurate node localization mostly depends on the delay in the signal through propagation medium and time delay in the circuits. These are the major contributor of the errors. The dramatic advances in IC design with low time delay have made possible to use these circuits in wireless sensor networks. Time delays in the circuits ranging from more than $1 \mathrm{~ns}$ down to the picoseconds range, depending on the technology being used. Signal propagation time delay which is the most crucial part in the measurements can be modeled as Gaussian with the following equation.

$$
f\left(T_{i, j}=t \mid \theta\right)=\aleph\left(t ; d_{i, j} / v_{p}+\mu T, \sigma_{T}^{2}\right)
$$

Where $\mu T$ and $\sigma_{T}^{2}$ are the mean and variance of the time delay error. UWB measurements conducted in Motorola factory showed $\mu T=0.3 \mathrm{~ns}$ and $\sigma_{T}=1.9 \mathrm{~ns}$. This mean error $\mu T$ can be estimated (as a nuisance parameter) by the localization algorithm so that it can be subtracted out [16]. But $\sigma_{T}$ has a great influence on the estimation of values. We have observed the error at different values of variance and compared the result with the synergetic localization algorithm [17]. With the ideal situation taking variance as low as 0.2 , we observed the average distance error of $0.11 \mathrm{~m}$ as shown in the Fig 6. In Fig 7 we have taken the variance as 2.0 and on this quantity we observed the average distance error of $0.71 \mathrm{~m}$.

The refinement process shown in Fig 8 tells that by increasing the number of iteration we get the lowest possible average distance error at different values of variance. It is true that with the increase of variance we lose accuracy of the system but the comparison results in Fig 9 shows that our system performs better at increased variance as compared to the synergetic localization algorithm.

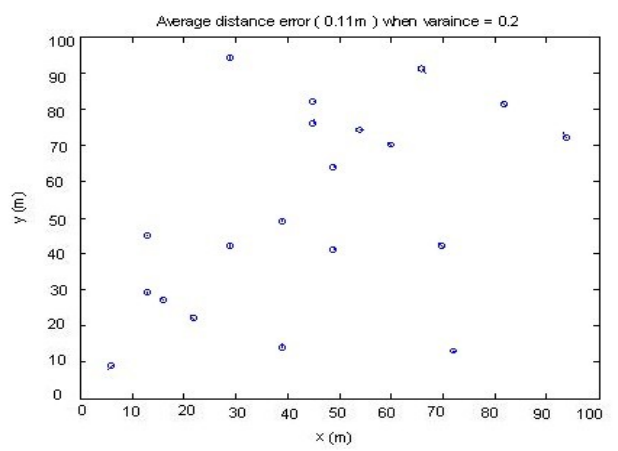

Figure 6. Average distance error with variance of 0.2

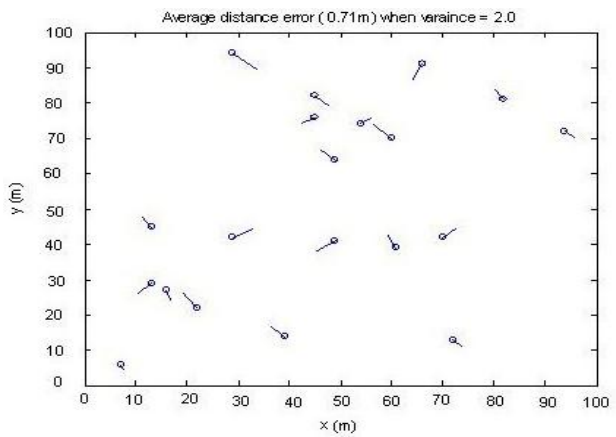

Figure 7. Average distance error with variance of 2.0

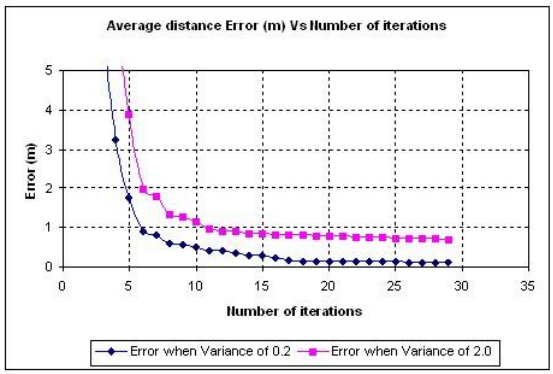

Figure 8. Average distance error Vs number of iteration 


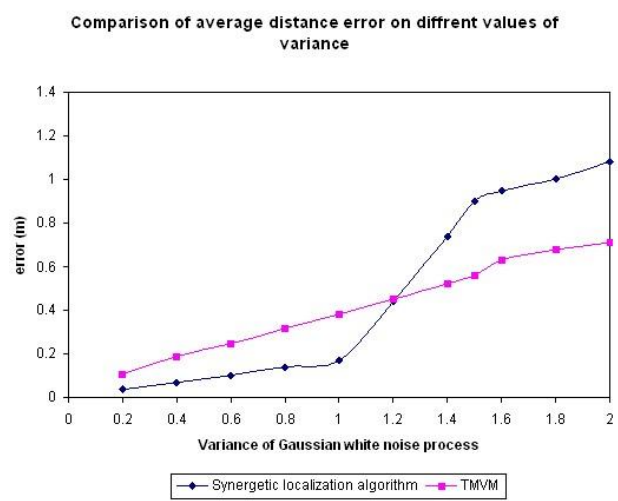

Figure 9. Comparison at diff values of variance

\section{CONCLUSION}

The usefulness of WSN operation mainly depends on the node location. Sensors can generally be placed in an area of interest either deterministically or randomly. The choice of the deployment scheme depends highly on the type of sensors, application and environment that the sensors will operate in. In this paper we addressed one of the crucial issues namely node localization by using Randomly Generated token with combination of Vector iterative process. This scheme gives more accuracy in terms of average distance error necessary for precise node localization. We get the close enough results after a number of refinement steps. This method behaves and provides the better performance on higher values of variance. This novel approach further strengthens its existence by dropping the dependency of central synchronized time reference necessary for node localization estimation.

\section{REFERENCES}

[1] I.F. Akyildiz, W. Su, Y. Sankarasubramaniam and E. Cayirci, Wireless sensor networks: a survey, Computer Networks 38 (2002), pp. 393-422.

[2] C-Y. Chong and S.P. Kumar, Sensor networks: evolution, opportunities, and challenges, Proceedings of the IEEE 91 (8) (2003), pp. 1247-1256.

[3] Pham Doan Tinh and Makoto Kawai, "Distributed Range-Free Localization Algorithm Based on Self-Organizing Maps," EURASIP Journal on Wireless Communications and Networking, vol. 2010, Article ID 692513, 9 pages, 2010. doi:10.1155/2010/ 692513

[4] D. Nicolescu and B. Nath, "Ad-hoc positioning systems (APS)," in Proceedings of IEEE Global Telecommunications Conference (GLOBECOM '01), San Antonio, Tex, USA, 2001.

[5] Y. Shang, W. Ruml, Y. Zhang, and M. Fromherz, "Localization from connectivity in sensor networks," IEEE Transactions on Parallel and Distributed Systems, vol. 15, no. 11, pp. 961-974, 2004.

[6] D. A. Tran and T. Nguyen, "Localization in wireless sensor networks based on support vector machines," IEEE Transactions on Parallel and Distributed Systems, vol. 19, no. 7, pp. 981-994, 2008.

[7] N. Patwari, A. O. Hero III, M. Perkins, N. S. Correal, and R. J. O'Dea, "Relative location estimation in wireless sensor networks," IEEE Transactions on Signal Processing, vol. 51, no.8, pp. 2137-2148, 2003
[8] Younis M., Akkaya K. Strategies and techniques for node placement in wireless sensor networks: A survey (2008) Ad Hoc Networks, 6 (4), pp. 621-655.

[9] D. Niculescu and B. Nath, "Ad hoc positioning system (APS) using AOA," in Proceedings of the 22nd Annual Joint Conference of the IEEE Computer and Communications Societies (INFOCOM '03), vol. 3, pp. 1734-1743, San Francisco, Calif, USA, 2003.

[10] X.Lu et al., Anti-localization anonymous routing for Delay Tolerant network, Compt.new. (2010), doi:10.1016/j.comnet.2010.03.002

[11] GuoQiang Mao \& Baris Fidan" Localization Algorithms and Strategies for Wireless Sensor Networks", Information Science Reference, Hershey, New York, 2009.

[12] T. He, C. Huang, B.M. Blum, J.A. Stankovic, T. Abdelzaher, Range-free localization schemes for large scale sensor networks, in Proceedings of the Nineth Annual International Conference on Mobile Computing and Networking (Mobicom'03), 2003, pp. 81-95.

[13] N. Bulusu, J. Heidemann and D. Estrin, GPS-less low cost outdoo localization for very small devices, IEEE Personal Communications 7 (5) (2000), pp. 28-34.

[14] L. Lazos and R. Poovendran, Serloc: secure range independent localization for wireless sensor networks, Proceedings of Third ACM Workshop on Wireless Security, ACM, Philadelphia, PA, USA (2004), pp. 21-30.

[15] H.-C. Chu and R.-H. Jan, A gps-less, outdoor, self-positioning method for wireless sensor networks, Ad Hoc Networks 5 (5) (2007), pp. $547-557$.

[16] N.S. Correal, S. Kyperountas, Q. Shi, and M. Welborn, "An ultra wideband relative location system," in Proc. IEEE Conf. Ultra Wideband Systems and Technologies, Nov. 2003, pp. 394-397.

[17] Jun Xiao, Hong Chen, Shi Zhang "Research of Range-based Synergetic Localization Algorithm in. Wireless Sensor Networks" CCDC 2008 